\title{
GROUP INVERSES FOR MATRICES OVER A BEZOUT DOMAIN*
}

\author{
CHONGGUANG $\mathrm{CAO}^{\dagger}$ AND JUYAN $\mathrm{LI}^{\dagger}$
}

\begin{abstract}
Suppose $\mathbf{R}$ is a Bezout domain. In this paper, some necessary and sufficient conditions for the existence of the group inverse for square matrix over $\mathbf{R}$ are given, the conditions for the existence of the group inverse of products of matrices are studied, and the equivalent conditions for reverse order law of group inverse of product of matrices are obtained. Also the existence and the representation of the group inverse for a class $2 \times 2$ block matrices over $\mathbf{R}$ are studied, and some well known relative results are generalized.
\end{abstract}

Key words. Bezout domain, Group inverse, Right $\mathbf{R}$-module, Block matrix.

AMS subject classifications. 15A09.

1. Introduction. Research on generalized inverses of matrices over commutative rings, especially the condition for the regularity of a matrix, is abundant; see e.g., [1] and references therein. However, research on the generalized inverses of matrices over non-commutative rings is relatively sparse; see [2], [4], [14], [15]. The purpose of this paper is to study the group inverses of matrices over an important associative ring - a Bezout domain, and generalize some results which are well known and given at present.

If every finitely generated left (right) ideal in a non-zero ring $\mathbf{R}$ which has a unit element 1 and no zero divisors is principal, then $\mathbf{R}$ is called a Bezout domain. Integral rings, non-commutative principal ideal domains, division rings, polynomial rings in an indeterminate over field, valuation rings and so on are Bezout domains; see [9], [11].

Let $\mathbf{R}$ be a Bezout domain, we denote the right $\mathbf{R}$-module of n-dimensional column vector and left $\mathbf{R}$-module of n-dimensional row vector by $\mathbf{R}_{r}^{n}$ and $\mathbf{R}_{l}^{n}$, respectively. Let $\mathbf{R}^{m \times n}$ be the set of all $m \times n$ matrices over $\mathbf{R}$. For a matrix $A \in \mathbf{R}^{n \times n}$, we denote the right $\mathbf{R}$-module which is generated by the columns, left $\mathbf{R}$-module which is generated by the rows, and right nullspace of $A$ by $R_{r}(A), R_{l}(A)$, and $N_{r}(A)$, respectively. The dimension of $R_{r}(A)$ and $R_{l}(A)$ is called the column rank and row

\footnotetext{
* Received by the editors June 4, 2009. Accepted for publication September 25, 2009. Handling Editor: Harm Bart.

${ }^{\dagger}$ Department of Mathematics, Heilongjiang University, Harbin, 150080, P. R. China (caochongguang@163.com, lijuyan587@163.com). Supported by Nation Science Foundation of China, No. 10671026.
} 
rank of $A$, respectively. It is well known that the row rank of $A$ is equal to its column rank, then their common value is called the rank of $A$, which is denoted by $\operatorname{rank} A$; see [9], [11]. A matrix is said to be nonsingular if it is neither 0 nor a left or right zero-divisor. We say that $A^{\#}$ is the group inverse of $A$ if $A^{\#}$ is a common solution of the matrix equations: $A X A=A, X A X=X, A X=X A$. It is well known that if $A^{\#}$ exists then $A^{\#}$ is unique.

We have two important results of matrices over $\mathbf{R}$, presented in the following lemmas; see [11].

Lemma 1.1. A ring $\mathbf{R}$ is a Bezout domain if and only if every non-zero matrix $A$ over $\mathbf{R}$ has a factorization $A=P\left[\begin{array}{ll}\Delta & 0 \\ 0 & 0\end{array}\right] Q$, where $P, Q$ are invertible matrices over $\mathbf{R}, \Delta \in \mathbf{R}^{k \times k}$ and $\operatorname{rank}(A)=k$.

Lemma 1.2. Let $\mathbf{R}$ be a Bezout domain, $A \in \mathbf{R}^{n \times n}$. Then $A$ is an idempotent matrix if and only if there exists invertible matrix $p \in \mathbf{R}^{n \times n}$ such that $A=$ $P\left[\begin{array}{ll}I_{r} & 0 \\ 0 & 0\end{array}\right] P^{-1}$.

This paper is divided into four sections. In the second section, we study the existence of the group inverse for matrix $A$, obtaining several equivalent conditions, as well as the existence and representation of the group inverse for $2 \times 2$ block upper triangular matrix. This generalizes the relative results in papers [4] and [13]. In the third section, we study the group inverses of products of two matrices, obtain the condition for the reverse order law of group inverses, and generalize the relative results in paper [5]. In the fourth section, we study the existence and representation of the group inverse for a class $2 \times 2$ block matrices, generalizing the relative results in paper [2]. All results we obtain are new even for commutative principal ideal domains.

\section{The Existence of the Group Inverse.}

Theorem 2.1. Suppose $A \in \mathbf{R}^{n \times n}$. Then the following conditions are equivalent:

(i) $A^{\#}$ exists .

(ii) $R_{r}(A)=R_{r}\left(A^{2}\right)$.

(iii) $R_{l}(A)=R_{l}\left(A^{2}\right)$.

(iv) There exist invertible matrices $D \in \mathbf{R}^{r \times r}$ and $N \in \mathbf{R}^{n \times n}$ such that $A=$ $N\left[\begin{array}{ll}D & 0 \\ 0 & 0\end{array}\right] N^{-1}$.

Proof. $(i) \Rightarrow$ (ii) It follows from the definition of the group inverse and $A^{\#}$ exists that $A=A^{2} A^{\#}$. Hence $R_{r}(A) \subseteq R_{r}\left(A^{2}\right)$. On the other hand $R_{r}(A) \supseteq R_{r}\left(A^{2}\right)$ is obvious. Then $R_{r}(A)=R_{r}\left(A^{2}\right)$. 
$(i) \Rightarrow($ iii $)$ The proof is similar to $(i) \Rightarrow($ ii $)$.

$(i v) \Rightarrow(i)$ Let $X=N\left[\begin{array}{ll}D^{-1} & 0 \\ 0 & 0\end{array}\right] N^{-1}$. It follows from the definition of the group inverse that $X=A^{\#}$.

(ii) $\Rightarrow\left(\right.$ iv) By Lemma 1.1, we can find invertible matrices $Q, P \in \mathbf{R}^{n \times n}$ such that $A=P\left[\begin{array}{cc}\Delta & 0 \\ 0 & 0\end{array}\right] Q$, where $\Delta$ is a $r \times r$ matrix with $\operatorname{rank} \Delta=r$. Since $R_{r}(A)=$ $R_{r}\left(A^{2}\right)$, we may write $A=A^{2} X$ for some matrix $X$. Let $Q P=\left[\begin{array}{ll}P_{1} & P_{2} \\ P_{3} & P_{4}\end{array}\right], X=$ $P\left[\begin{array}{cc}X_{1} & X_{2} \\ X_{3} & X_{4}\end{array}\right] P^{-1}$, where $P_{1}, X_{1} \in \mathbf{R}^{r \times r}$. Then

$$
\begin{gathered}
A=P\left[\begin{array}{lr}
\Delta P_{1} & \Delta P_{2} \\
0 & 0
\end{array}\right] P^{-1}, \text { and } \\
A^{2} X=P\left[\begin{array}{lr}
\Delta P_{1} \Delta P_{1} X_{1}+\Delta P_{1} \Delta P_{2} X_{3} & \Delta P_{1} \Delta P_{1} X_{2}+\Delta P_{1} \Delta P_{2} X_{4} \\
0 & 0
\end{array}\right] P^{-1} .
\end{gathered}
$$

Hence

$$
\begin{gathered}
\Delta P_{1} \Delta P_{1} X_{1}+\Delta P_{1} \Delta P_{2} X_{3}=\Delta P_{1}, \Delta P_{1} \Delta P_{1} X_{2}+\Delta P_{1} \Delta P_{2} X_{4}=\Delta P_{2}, \\
P_{1} \Delta P_{1} X_{1}+P_{1} \Delta P_{2} X_{3}=P_{1}, \text { and } P_{1} \Delta P_{1} X_{2}+P_{1} \Delta P_{2} X_{4}=P_{2} .
\end{gathered}
$$

It follows that there exists matrix $Z$ such that $\left[\begin{array}{ll}P_{1} & P_{2}\end{array}\right] Z=I$ from invertibility of the matrices $Q P$, then

$$
\begin{gathered}
{\left[\begin{array}{cc}
P_{1} \Delta P_{1} X_{1}+P_{1} \Delta P_{2} X_{3} & P_{1} \Delta P_{1} X_{2}+P_{1} \Delta P_{2} X_{4}
\end{array}\right] Z=I, \text { and }} \\
P_{1} \Delta\left[\begin{array}{ll}
P_{1} X_{1}+P_{2} X_{3} & P_{1} X_{2}+P_{2} X_{4}
\end{array}\right] Z=I .
\end{gathered}
$$

Hence $P_{1}$ and $\Delta$ are invertible matrices. Namely $\Delta P_{1}$ is an invertible matrix. Let $N=P\left[\begin{array}{rr}I & -P_{1}^{-1} P_{2} \\ 0 & I\end{array}\right]$ and $D=\Delta P_{1}$, then $A=N\left[\begin{array}{ll}D & 0 \\ 0 & 0\end{array}\right] N^{-1}$.

$($ iii $) \Rightarrow($ iv $)$ The proof is similar to $(i i) \Rightarrow(i v)$.

REMARK 2.2. If $\mathbf{R}$ is a skew field, then $R_{r}(A)=R_{r}\left(A^{2}\right)$ if and only if $\operatorname{rank}(A)=$ $\operatorname{rank}\left(A^{2}\right)$. However, if $\mathbf{R}$ is a general Bezout domain, then $R_{r}(A)=R_{r}\left(A^{2}\right)$ and $\operatorname{rank}(A)=\operatorname{rank}\left(A^{2}\right)$ are not equivalent, e.g. if $\mathbf{R}$ is a ring of integral number, let $A=\left[\begin{array}{ll}1 & 1 \\ 1 & 1\end{array}\right]$, then $A^{2}=\left[\begin{array}{ll}2 & 2 \\ 2 & 2\end{array}\right]$. It is obvious that $\operatorname{rank}(A)=\operatorname{rank}\left(A^{2}\right)$, but $R_{r}(A) \neq R_{r}\left(A^{2}\right)$. 
Theorem 2.3. Suppose $M=\left[\begin{array}{cc}A & B \\ 0 & C\end{array}\right] \in \mathbf{R}^{n \times n}, A \in \mathbf{R}^{m \times m}$. Then:

(1) $M^{\#}$ exists if and only if $A^{\#}$, and $C^{\#}$ exist and $\left(I-A A^{\#}\right) B\left(I-C C^{\#}\right)=0$.

(2) If $M^{\#}$ exists, then $M^{\#}=\left[\begin{array}{lr}A^{\#} & X \\ 0 & C^{\#}\end{array}\right]$, where $X=\left(A^{\#}\right)^{2} B\left(I-C C^{\#}\right)+$ $\left(I-A A^{\#}\right) B\left(C^{\#}\right)^{2}-A^{\#} B C^{\#}$.

Proof. (1) ('if') Let $Y=\left[\begin{array}{lr}A^{\#} & X \\ 0 & C^{\#}\end{array}\right]$, where $X=\left(A^{\#}\right)^{2} B\left(I-C C^{\#}\right)+(I-$ $\left.A A^{\#}\right) B\left(C^{\#}\right)^{2}-A^{\#} B C^{\#}$. It follows from the existence of $A^{\#}$ and $C^{\#}$, and $(I-$ $\left.A A^{\#}\right) B\left(I-C C^{\#}\right)=0$ that $M Y M=M, Y M Y=Y$, and $M Y=Y M$. Therefore $M^{\#}$ exists.

('only if') It follows from the existence of $M^{\#}$ and Theorem 2.1(ii) that $R_{r}(M)=$ $R_{r}\left(M^{2}\right)$. Thus, for any $x_{1} \in \mathbf{R}_{r}^{m}, x_{2} \in \mathbf{R}_{r}^{n-m}$, there exists $y_{1} \in \mathbf{R}_{r}^{m}, y_{2} \in \mathbf{R}_{r}^{n-m}$, such that:

$$
\left[\begin{array}{ll}
A & B \\
0 & C
\end{array}\right]\left[\begin{array}{l}
x_{1} \\
x_{2}
\end{array}\right]=\left[\begin{array}{lr}
A^{2} & A B+B C \\
0 & C^{2}
\end{array}\right]\left[\begin{array}{l}
y_{1} \\
y_{2}
\end{array}\right] .
$$

Hence $C x_{2}=C^{2} y_{2}$. This means $R_{r}(C) \subseteq R_{r}\left(C^{2}\right) . \quad R_{r}(C) \supseteq R_{r}\left(C^{2}\right)$ is obvious. Hence $R_{r}(C)=R_{r}\left(C^{2}\right)$. Again applying Theorem 2.1(ii), we know $C^{\#}$ exists. By Theorem 2.1(iii), for any $u_{1} \in \mathbf{R}_{l}^{m}, u_{2} \in \mathbf{R}_{l}^{n-m}$ there exists $z_{1} \in \mathbf{R}_{l}^{m}, z_{2} \in \mathbf{R}_{l}^{n-m}$ such that

$$
\left[\begin{array}{ll}
u_{1} & u_{2}
\end{array}\right]\left[\begin{array}{ll}
A & B \\
0 & C
\end{array}\right]=\left[\begin{array}{ll}
z_{1} & z_{2}
\end{array}\right]\left[\begin{array}{lr}
A^{2} & A B+B C \\
0 & C^{2}
\end{array}\right] .
$$

Hence $u_{1} A=z_{1} A^{2}$. This means $R_{l}(A)=R_{l}\left(A^{2}\right)$, that is $A^{\#}$ exists. It follows from Theorem 2.1(iv) that $A=P\left[\begin{array}{ll}D_{1} & 0 \\ 0 & 0\end{array}\right] P^{-1}$ and $C=Q^{-1}\left[\begin{array}{ll}D_{2} & 0 \\ 0 & 0\end{array}\right] Q$, where $D_{1} \in \mathbf{R}^{s \times s}$ and $D_{2} \in \mathbf{R}^{t \times t}$ are invertible matrices. Let

$$
P^{-1} B Q^{-1}=\left[\begin{array}{cc}
B_{1} & B_{2} \\
B_{3} & B_{4}
\end{array}\right], B_{4} \in \mathbf{R}^{(m-s) \times(n-m-t)} .
$$

It is easy to see

$$
\left(I-A A^{\#}\right) B\left(I-C C^{\#}\right)=P\left[\begin{array}{ll}
0 & 0 \\
0 & I
\end{array}\right] P^{-1} B Q^{-1}\left[\begin{array}{cc}
0 & 0 \\
0 & I
\end{array}\right] Q=P\left[\begin{array}{rr}
0 & 0 \\
0 & B_{4}
\end{array}\right] Q .
$$

In order to prove the conclusion, we only need to prove $B_{4}=0$. In fact,

$$
M=\left[\begin{array}{ll}
A & B \\
0 & C
\end{array}\right]=\left[\begin{array}{rr}
P & 0 \\
0 & Q^{-1}
\end{array}\right]\left[\begin{array}{cccc}
D_{1} & 0 & B_{1} & B_{2} \\
0 & 0 & B_{3} & B_{4} \\
0 & 0 & D_{2} & 0 \\
0 & 0 & 0 & 0
\end{array}\right]\left[\begin{array}{cc}
P^{-1} & 0 \\
0 & Q
\end{array}\right]
$$


¿From it we know $M \sim\left[\begin{array}{cccc}D_{1} & 0 & B_{1} & 0 \\ 0 & 0 & 0 & B_{4} \\ 0 & 0 & D_{2} & 0 \\ 0 & 0 & 0 & 0\end{array}\right]$. Since $M^{\#}$ exists, we have $N^{\#}=$ $\left[\begin{array}{rr}0 & B_{4} \\ 0 & 0\end{array}\right]^{\#}$. Obviously, $N^{2}=0$. Then $N=N^{2} N^{\#}=0$, and $B_{4}=0$.

(2) It is obvious from the proof of (1).

Corollary 2.4. Suppose $B_{1} \in \mathbf{R}^{m \times m}$ and $M=\left[\begin{array}{cr}B_{1} & B_{2} \\ 0 & 0\end{array}\right] \in \mathbf{R}^{n \times n}$. Then

(1) $M^{\#}$ exists if and only if $B_{1}^{\#}$ exists and $R_{r}\left(B_{2}\right) \subset R_{r}\left(B_{1}\right)$.

(2) If $M^{\#}$ exists, then $M^{\#}=\left[\begin{array}{lr}B_{1}^{\#} & \left(B_{1}^{\#}\right)^{2} B_{2} \\ 0 & 0\end{array}\right]$.

Proof. By Theorem 2.3(2), let $C=0$. The result follows easily.

Corollary 2.5. Suppose $B_{1} \in \mathbf{R}^{m \times m}$ and $M=\left[\begin{array}{cc}B_{1} & 0 \\ B_{2} & 0\end{array}\right] \in \mathbf{R}^{n \times n}$. Then

(1) $M^{\#}$ exists if and only if $B_{1}^{\#}$ exists and $R_{l}\left(B_{2}\right) \subset R_{l}\left(B_{1}\right)$.

(2) If $M^{\#}$ exists, then $M^{\#}=\left[\begin{array}{ll}B_{1}^{\#} & 0 \\ B_{2}\left(B_{1}^{\#}\right)^{2} & 0\end{array}\right]$.

Proof. We can get the formula for the group inverse of $\left[\begin{array}{cc}A & 0 \\ B & C\end{array}\right]$ by the same proof of Theorem 2.3. Let $C=0$. The result follows easily. $\square$

\section{Group Inverses of Products of Two Matrices.}

Theorem 3.1. Suppose $A \in \mathbf{R}^{m \times n}, B \in \mathbf{R}^{n \times m}$. Then the following conditions are equivalent:

(1) $(A B)^{\#}$ and $(B A)^{\#}$ exist.

(2) $R_{r}(A B)=R_{r}(A B A)$ and $R_{r}(B A)=R_{r}(B A B)$.

(3) $R_{l}(A B)=R_{l}(A B A)$ and $R_{l}(B A)=R_{l}(B A B)$.

Proof. (1) $\Rightarrow(2)$ It follows from the existence of $(A B)^{\#}$ and Theorem2.1 that $R_{r}(A B)=R_{r}(A B A B) . \quad R_{r}(A B A B) \subseteq R_{r}(A B A) \subseteq R_{r}(A B)$ is obvious. Thus $R_{r}(A B)=R_{r}(A B A)$. Similarly $R_{r}(B A)=R_{r}(B A B)$.

$(2) \Rightarrow(1)$ From $R_{r}(A B)=R_{r}(A B A)=A R_{r}(B A)$ and $R_{r}(B A)=R_{r}(B A B)$, we have $R_{r}(A B)=R_{r}(A B A)=R_{r}(A B A B)$. Thus $(A B)^{\#}$ exists. Similarly $(B A)^{\#}$ exists. 
$(1) \Leftrightarrow(3)$ The proof is similar to $(1) \Leftrightarrow(2)$.

Corollary 3.2. Suppose $A \in \mathbf{R}^{m \times n}, B \in \mathbf{R}^{n \times m}$, and $R_{r}(A)=R_{r}(A B A)$, then $(A B)^{\#}$ and $(B A)^{\#}$ exist.

Proof. Since $R_{r}(A B A) \subseteq R_{r}(A B) \subseteq R_{r}(A)$, and $R_{r}(A)=R_{r}(A B A)$, we have $R_{r}(A B A)=R_{r}(A B)=R_{r}(A)$. Thus $R_{r}(A B)=R_{r}(A B A)$. By $R_{r}(A B A)=R_{r}(A)$, we have $R_{r}(B A B A)=R_{r}(B A)$. Thus $R_{r}(B A)=R_{r}(B A B)$. From Theorem 3.1, we can prove immediately. $\square$

Corollary 3.3. Suppose $A \in \mathbf{R}^{m \times n}, B \in \mathbf{R}^{n \times m}$, and $R_{r}(A B)=R_{r}(A)$, $R_{r}(B A)=R_{r}(B)$, then $(A B)^{\#}$ and $(B A)^{\#}$ exist.

Proof. It follows from $R_{r}(A B)=R_{r}(A)$, and $R_{r}(B A)=R_{r}(B)$ that $R_{r}(A B)=$ $A R_{r}(B)=A R_{r}(B A)=R_{r}(A B A)$. Similarly, $R_{r}(B A)=R_{r}(B A B)$. ¿From Theorem 3.1 , we can prove immediately.

Corollary 3.4. Suppose $A \in \mathbf{R}^{m \times n}, B \in \mathbf{R}^{n \times m}, R_{r}(A B)=R_{r}(A)$ and $(A B)^{\#}$ exist, then $(B A)^{\#}$ exist.

Proof. It follows from the existence of $(A B)^{\#}$ that $R_{r}(A B)=R_{r}(A B A B)$. Hence $R_{r}(A B)=R_{r}(A B A)$. From $R_{r}(A B)=R_{r}(A)$ it follows that $B R_{r}(A B)=B R_{r}(A)$. Therefore $R_{r}(B A)=R_{r}(B A B)$. Then we can obtain the existence of $(B A)^{\#}$ from Theorem 3.1 .

Lemma 3.5. Suppose $A \in \mathbf{R}^{m \times n}, B \in \mathbf{R}^{n \times m}$, then there exist invertible matrices $P \in \mathbf{R}^{m \times m}$ and $Q \in \mathbf{R}^{n \times n}$, such that

$$
A=P\left[\begin{array}{ll}
A_{1} & 0 \\
0 & 0
\end{array}\right] Q \text {, and } B=Q^{-1}\left[\begin{array}{ccc}
B_{1} & B_{2} & B_{3} \\
0 & 0_{r-s} & 0 \\
C_{1} & C_{2} & C_{3} \\
0 & 0 & 0
\end{array}\right] P^{-1} \text {, }
$$

where $A_{1} \in \mathbf{R}^{r \times r}, \operatorname{rank} A=\operatorname{rank} A_{1}=r, B_{1} \in \mathbf{R}^{s \times s}, C_{1} \in \mathbf{R}^{t \times s}$.

Proof. By Lemma 1.1, we can find invertible matrices $M \in \mathbf{R}^{m \times m}, N \in \mathbf{R}^{n \times n}$ such that $A=M\left[\begin{array}{cc}\Delta & 0 \\ 0 & 0\end{array}\right] N$, where $\Delta \in \mathbf{R}^{r \times r}$ and $\operatorname{rank} A=r$. We write $B=$ $N^{-1}\left[\begin{array}{c}\Delta_{1} \\ \Delta_{2}\end{array}\right] M^{-1}$, where $\Delta_{1} \in \mathbf{R}^{r \times m}, \Delta_{1}=Q_{1}^{-1}\left[\begin{array}{cc}E & 0 \\ 0 & 0\end{array}\right] P_{1}^{-1}$, and $E$ is a $s \times s$ non-singular matrix over R. $\Delta_{2}=Q_{2}^{-1}\left[\begin{array}{ll}F & 0 \\ 0 & 0\end{array}\right] P_{2}^{-1}$, where $F$ is a $t \times t$ non-singular matrix over $\mathbf{R}$. Let

$$
\left[\begin{array}{ll}
E & 0 \\
0 & 0
\end{array}\right] P_{1}^{-1}=\left[\begin{array}{ccc}
B_{1} & B_{2} & B_{3} \\
0 & 0_{r-s} & 0
\end{array}\right] \text {, and }\left[\begin{array}{cc}
F & 0 \\
0 & 0
\end{array}\right] P_{2}^{-1}=\left[\begin{array}{ccc}
C_{1} & C_{2} & C_{3} \\
0 & 0 & 0
\end{array}\right]
$$


where $B_{1} \in \mathbf{R}^{s \times s}$, and $C_{1} \in \mathbf{R}^{t \times s}$. Then

$$
\begin{aligned}
& B=N^{-1}\left[\begin{array}{lr}
Q_{1}^{-1} & 0 \\
0 & Q_{2}^{-1}
\end{array}\right]\left[\begin{array}{ccc}
B_{1} & B_{2} & B_{3} \\
0 & 0_{r-s} & 0 \\
C_{1} & C_{2} & C_{3} \\
0 & 0 & 0
\end{array}\right] M^{-1} \text {, and } \\
& A=M\left[\begin{array}{ll}
\Delta Q_{1}^{-1} & 0 \\
0 & 0
\end{array}\right]\left[\begin{array}{lr}
Q_{1} & 0 \\
0 & Q_{2}
\end{array}\right] N .
\end{aligned}
$$

Let $M=P,\left[\begin{array}{cr}Q_{1} & 0 \\ 0 & Q_{2}\end{array}\right] N=Q$, and $\Delta Q_{1}^{-1}=A_{1}$, then $A_{1} \in \mathbf{R}^{r \times r}, \operatorname{rank} A=$ $\operatorname{rank} A_{1}=r$, and

$$
A=P\left[\begin{array}{ll}
A_{1} & 0 \\
0 & 0
\end{array}\right] \text {, and } B=Q^{-1}\left[\begin{array}{ccc}
B_{1} & B_{2} & B_{3} \\
0 & 0_{r-s} & 0 \\
C_{1} & C_{2} & C_{3} \\
0 & 0 & 0
\end{array}\right] P^{-1} \text {. }
$$

Theorem 3.6. Suppose $A, B \in \mathbf{R}^{n \times n}$. Then from any two of the following conditions, we can obtain the other one.

(1) $(A B)^{\#}$ exists.

(2) $(B A)^{\#}$ exists.

(3) $A B \sim B A$.

Proof. (1), (2) $\Rightarrow(3)$ Let $A$ and $B$ be the form as in Lemma 3.5, then

$$
\begin{aligned}
& A B=P\left[\begin{array}{l}
A_{1}\left[\begin{array}{rr}
B_{1} & B_{2} \\
0 & 0
\end{array}\right] \quad A_{1}\left[\begin{array}{l}
B_{3} \\
0
\end{array}\right] \\
0
\end{array}\right] P^{-1}, \text { and }
\end{aligned}
$$

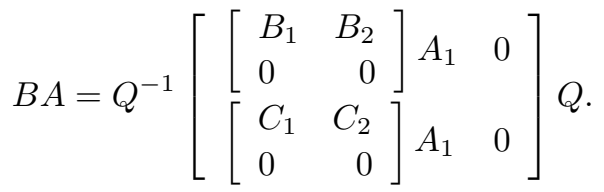

¿From Corollary 2.4 and Corollary 2.5, we obtain the following:

$$
\begin{gathered}
(A B)^{\#} \text { exists } \Leftrightarrow\left(A_{1}\left[\begin{array}{rr}
B_{1} & B_{2} \\
0 & 0
\end{array}\right]\right) \# \text { exists, and } \\
R_{r}\left(A_{1}\left[\begin{array}{l}
B_{3} \\
0
\end{array}\right]\right) \subset R_{r}\left(A_{1}\left[\begin{array}{rr}
B_{1} & B_{2} \\
0 & 0
\end{array}\right]\right),
\end{gathered}
$$




$$
\begin{gathered}
(B A)^{\#} \text { exists } \Leftrightarrow\left(\left[\begin{array}{rr}
B_{1} & B_{2} \\
0 & 0
\end{array}\right] A_{1}\right)^{\#} \text { exists, and } \\
R_{l}\left(\left[\begin{array}{rr}
C_{1} & C_{2} \\
0 & 0
\end{array}\right] A_{1}\right) \subset R_{l}\left(\left[\begin{array}{rr}
B_{1} & B_{2} \\
0 & 0
\end{array}\right] A_{1}\right) .
\end{gathered}
$$

Hence there exists $E \in \mathbf{R}^{r \times(n-r)}, F \in \mathbf{R}^{(n-r) \times r}$ such that

$$
A_{1}\left[\begin{array}{l}
B_{3} \\
0
\end{array}\right]=A_{1}\left[\begin{array}{rr}
B_{1} & B_{2} \\
0 & 0
\end{array}\right] E \text {, and }\left[\begin{array}{lr}
C_{1} & C_{2} \\
0 & 0
\end{array}\right] A_{1}=F\left[\begin{array}{lr}
B_{1} & B_{2} \\
0 & 0
\end{array}\right] A_{1} .
$$

Then

$$
\begin{aligned}
& A B=P\left[\begin{array}{rr}
I & -E \\
0 & I
\end{array}\right]\left[\begin{array}{l}
A_{1}\left[\begin{array}{rr}
B_{1} & B_{2} \\
0 & 0
\end{array}\right] \\
0
\end{array}\right.
\end{aligned}
$$

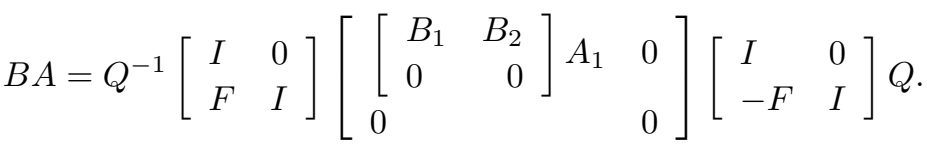

Let $\left[\begin{array}{cr}B_{1} & B_{2} \\ 0 & 0\end{array}\right]=R\left[\begin{array}{cc}D & 0 \\ 0 & 0\end{array}\right] S$, for invertible matrices $R, S$, where $D \in \mathbf{R}^{r_{1} \times r_{1}}$ and $\operatorname{rank} D=r_{1}$. Then

$$
A_{1}\left[\begin{array}{cr}
B_{1} & B_{2} \\
0 & 0
\end{array}\right] \sim S A_{1} R\left[\begin{array}{ll}
D & 0 \\
0 & 0
\end{array}\right] \text {, and }\left[\begin{array}{cr}
B_{1} & B_{2} \\
0 & 0
\end{array}\right] A_{1} \sim\left[\begin{array}{cc}
D & 0 \\
0 & 0
\end{array}\right] S A_{1} R .
$$

Let $C=S A_{1} R, C=\left[\begin{array}{cc}C_{4} & C_{5} \\ C_{6} & C_{7}\end{array}\right]$. Then $\operatorname{rank} C=r$,

$$
C\left[\begin{array}{ll}
D & 0 \\
0 & 0
\end{array}\right]=\left[\begin{array}{ll}
C_{4} D & 0 \\
C_{6} D & 0
\end{array}\right] \text {, and }\left[\begin{array}{ll}
D & 0 \\
0 & 0
\end{array}\right] C=\left[\begin{array}{lr}
D C_{4} & D C_{5} \\
0 & 0
\end{array}\right]
$$

It follows from $\left(A_{1}\left[\begin{array}{rr}B_{1} & B_{2} \\ 0 & 0\end{array}\right]\right)^{\#}$ exists that $\left(C\left[\begin{array}{ll}D & 0 \\ 0 & 0\end{array}\right]\right)^{\#}$ exist, then $\left(C_{4} D\right)^{\#}$ exists, and $R_{l}\left(C_{6} D\right) \subset R_{l}\left(C_{4} D\right)$, then $C_{6} D=G C_{4} D$ for some matrix $G$. Hence

$$
C\left[\begin{array}{ll}
D & 0 \\
0 & 0
\end{array}\right] \sim\left[\begin{array}{ll}
C_{4} D & 0 \\
0 & 0
\end{array}\right] \text {, and } \operatorname{rank}\left(C_{4} D\right)=r_{1} .
$$

Similarly, we can prove $\left(D C_{4}\right)^{\#}$ exists,

$$
\left[\begin{array}{ll}
D & 0 \\
0 & 0
\end{array}\right] C \sim\left[\begin{array}{ll}
D C_{4} & 0 \\
0 & 0
\end{array}\right] \text {, and } \operatorname{rank}\left(D C_{4}\right)=r_{1} .
$$

It follows from the definition of the group inverse and the existence of $\left(C_{4} D\right)^{\#}$. Thus 


$$
C_{4} D X C_{4} D=C_{4} D \Rightarrow C_{4} D\left(X C_{4} D-I\right)=0 \Rightarrow X C_{4} D-I=0 \Rightarrow X C_{4} D=I
$$

for matrix a $X$. Hence $C_{4}$, and $D$ are invertible matrices, and $C_{4} D \sim D C_{4}$. Therefore $A B \sim B A$.

$(1),(3) \Rightarrow(2)$ and $(2),(3) \Rightarrow(1)$ are obvious.

Theorem 3.7. Suppose $A, B \in \mathbf{R}^{n \times n}$, and $A^{\#}$ and $B^{\#}$ exist. Then the following conditions are equivalent:

(i) $(A B)^{\#}$ exists and $(A B)^{\#}=B^{\#} A^{\#}$.

(ii) $R_{r}(A B)=R_{r}(B A)$ and $N_{r}(A B)=N_{r}(B A)$.

(iii) There exists a invertible $P \in \mathbf{R}^{n \times n}$ such that

$$
\left\{\begin{array}{l}
A=P\left(A_{1} \oplus \cdots \oplus A_{t}\right) P^{-1} \\
B=P\left(B_{1} \oplus \cdots \oplus B_{t}\right) P^{-1}
\end{array}\right.
$$

where $A_{i}=0$ or $A_{i}$ is invertible for every $i, B_{i}=0$ or $B_{i}$ is invertible for every $i$, the orders of $A_{i}$ and $B_{i}$ are equal for every $i$, and $A_{j} B_{j}=0$ for $j \geq$ 2.

Proof. $\quad(i i i) \Rightarrow(i)$ and $($ iii $) \Rightarrow($ ii $)$ can be obtained by a direct computation.

(i) $\Rightarrow$ (iii) We proceed by induction on $n$. If $n=1$, the proof is obvious. Suppose the lemma is true when $k<n$, where $k \geq 2$; we will prove that it is true when $k=n$. Without loss of generality, we assume $0<\operatorname{rank} A<n$. It follows from Theorem 2.1(iv) and the existence of $A^{\#}$ that

$$
A=N\left[\begin{array}{ll}
D & 0 \\
0 & 0
\end{array}\right] N^{-1} \text {, and } A^{\#}=N\left[\begin{array}{ll}
D^{-1} & 0 \\
0 & 0
\end{array}\right] N^{-1}
$$

for some invertible matrices $N \in \mathbf{R}^{n \times n}, D \in \mathbf{R}^{r \times r}$, where $r=\operatorname{rank} A$. Let

$$
B=N\left[\begin{array}{ll}
B_{1} & B_{2} \\
B_{3} & B_{4}
\end{array}\right] N^{-1} \text {, and } B^{\#}=N\left[\begin{array}{ll}
C_{1} & C_{2} \\
C_{3} & C_{4}
\end{array}\right] N^{-1}
$$

where $B_{1}, C_{1} \in \mathbf{R}^{r \times r}$. Then

$$
A B=N\left[\begin{array}{lr}
D B_{1} & D B_{2} \\
0 & 0
\end{array}\right] N^{-1}, \text { and } B^{\#} A^{\#}=N\left[\begin{array}{ll}
C_{1} D^{-1} & 0 \\
C_{3} D^{-1} & 0
\end{array}\right] N^{-1} .
$$

By Corollary 2.4 and $(A B)^{\#}=B^{\#} A^{\#}$, we have

$$
\left[\left(D B_{1}\right)^{\#}\right]^{2} D B_{2}=0 \text {, and } C_{3}=0 .
$$

Again applying Corollary 2.4 and the existence of $(A B)^{\#}$, we have $R_{r}\left(D B_{2}\right) \subset$ $R_{r}\left(D B_{1}\right)$. Thus, $D B_{2}=D B_{1} X$ for some $r \times(n-r)$ matrix X, i.e., $B_{2}=B_{1} X$. Replacing $B_{2}$ by $B_{1} X$ in (3.3), we have $D B_{1} X=0$. Hence

$$
B_{2}=D^{-1}\left(D B_{2}\right)=D^{-1}\left(D B_{1} X\right)=0
$$


Combining (3.2), (3.3), and (3.4), we have $\left[\begin{array}{rr}B_{1} & 0 \\ B_{3} & B_{4}\end{array}\right]=\left[\begin{array}{ll}C_{1} & C_{2} \\ 0 & C_{4}\end{array}\right]^{\#}$. It follows from Theorem 2.3 that $B_{3}=0$. In summary,

$$
A=N(D \oplus 0) N^{-1}, \text { and } B=N\left(B_{1} \oplus B_{4}\right) N^{-1}
$$

Again applying $(A B)^{\#}=B^{\#} A^{\#}$, we obtain $\left(D B_{1}\right)^{\#}=B_{1}^{\#} D^{\#}$, and $\left(0 B_{4}\right)^{\#}=B_{4}^{\#} 0$. By the induction hypothesis, it is easy to see that (iii) holds.

(ii) $\Rightarrow($ iii $)$ We proceed by induction on $n$. If $n=1$, the proof is obvious. Suppose the lemma is true when $k<n$, where $k \geq 2$; we will prove that it is true when $k=n$. We can assume that (3.1) and (3.2) hold. It follows from $R_{r}(A B)=$ $R_{r}(B A)$ that $B_{3} D=0$, i.e., $B_{3}=0$. Again applying $N_{r}(A B)=N_{r}(B A)$, we have $N_{r}\left(\left[\begin{array}{lr}D B_{1} & D B_{2} \\ 0 & 0\end{array}\right]\right)=N_{r}\left(\left[\begin{array}{ll}B_{1} D & 0 \\ 0 & 0\end{array}\right]\right)$. Noting $\left[\begin{array}{l}0 \\ y\end{array}\right] \in N_{r}\left(\left[\begin{array}{ll}B_{1} D & 0 \\ 0 & 0\end{array}\right]\right)$ for any $y \in \mathbf{C}^{n-r}$, we obtain $\left[\begin{array}{l}0 \\ y\end{array}\right] \in N_{r}\left(\left[\begin{array}{cr}D B_{1} & D B_{2} \\ 0 & 0\end{array}\right]\right)$, and hence $D B_{2} y=0$. That is $B_{2}=0$. In summary Equation (3.5) holds. Again applying (ii), we obtain that $R_{r}\left(D B_{1}\right)=R_{r}\left(B_{1} D\right), N_{r}\left(D B_{1}\right)=N_{r}\left(B_{1} D\right), R_{r}\left(0 B_{4}\right)=R_{r}\left(B_{4} 0\right)$, and $N_{r}\left(0 B_{4}\right)=$ $N_{r}\left(B_{4} 0\right)$. By the induction hypothesis, it is easy to see that (iii) holds.

Corollary 3.8. Suppose $A, B \in \mathbf{R}^{n \times n}, A^{\#}$ and $B^{\#}$ exist, and $A B=B A$. Then $(A B)^{\#}$ exists and $(A B)^{\#}=B^{\#} A^{\#}$.

Corollary 3.9. Suppose $A, B \in \mathbf{R}^{n \times n}, A^{\#}$ and $B^{\#}$ exist. Then $(A B)^{\#}$ exists and $(A B)^{\#}=B^{\#} A^{\#}$ if and only if $(B A)^{\#}$ exists and $(B A)^{\#}=A^{\#} B^{\#}$.

4. Group Inverse for a Class $2 \times 2$ Block Matrices. The group inverses of block matrices have various applications in singular differential and difference equations, Markov chains, iterative methods; see [1], [3], [6], [7], [8], [10], [12], [13], [16], [17]. We generalized the results of [2] to the Bezout domain in this section of the paper.

Lemma 4.1. Let $A, B \in \mathbf{R}^{n \times n}$, if $A^{2}=A, \operatorname{rank} A=r, R_{r}(B)=R_{r}(B A B)$, then there is an invertible matrix $P \in \mathbf{R}^{n \times n}$, such that $B=P\left[\begin{array}{cc}B_{1} & B_{1} X \\ Y B_{1} & Y B_{1} X\end{array}\right] P^{-1}$ and $B_{1}^{\#}$ exists, where $B_{1} \in \mathbf{R}^{r \times r}, X \in \mathbf{R}^{r \times(n-r)}, Y \in \mathbf{R}^{(n-r) \times r}$.

Proof. Since $A^{2}=A$, by Lemma 1.2 there exists invertible matrix $P \in \mathbf{R}^{n \times n}$ such that $A=P\left[\begin{array}{ll}I_{r} & 0 \\ 0 & 0\end{array}\right] P^{-1}, B=P\left[\begin{array}{cc}B_{1} & B_{2} \\ B_{3} & B_{4}\end{array}\right] P^{-1}$, where $B_{1} \in \mathbf{R}^{r \times r}, B_{4} \in$ $\mathbf{R}^{(n-r) \times(n-r)}$. It follows from $R_{r}(B)=R_{r}(B A B)$ that $R_{r}(B)=R_{r}(B A)$. Then there exists $Z=P\left[\begin{array}{cc}Z_{1} & X \\ Z_{3} & Z_{4}\end{array}\right] P^{-1} \in \mathbf{R}^{n \times n}$, where $X \in \mathbf{R}^{r \times(n-r)}$, such that $B=B A Z$, 
i.e.,

$$
\left[\begin{array}{ll}
B_{1} & B_{2} \\
B_{3} & B_{4}
\end{array}\right]=\left[\begin{array}{ll}
B_{1} & 0 \\
B_{3} & 0
\end{array}\right]\left[\begin{array}{cc}
Z_{1} & X \\
Z_{3} & Z_{4}
\end{array}\right]=\left[\begin{array}{ll}
B_{1} Z_{1} & B_{1} X \\
B_{3} Z_{1} & B_{3} X
\end{array}\right]
$$

then $B_{2}=B_{1} X$, and $B_{4}=B_{3} X$. It follows from $R_{r}(B)=R_{r}(B A B)$ and Corollary 3.2 that $(B A)^{\#}$ exists. By Corollary 2.5 and $B A=P\left[\begin{array}{cc}B_{1} & 0 \\ B_{3} & 0\end{array}\right] P^{-1}$, we get that $B_{1}^{\#}$ exists and $R_{l}\left(B_{3}\right) \subset R_{l}\left(B_{1}\right)$. So there is a matrix $Y \in \mathbf{R}^{(n-r) \times r}$, such that $B_{3}=Y B_{1}$. That is $B=P\left[\begin{array}{lr}B_{1} & B_{1} X \\ Y B_{1} & Y B_{1} X\end{array}\right] P^{-1}$.

Lemma 4.2. Let $A, B \in \mathbf{R}^{n \times n}$. If $A^{2}=A, \operatorname{rank} A=r, R_{r}(B)=R_{r}(B A B)$, then the following conclusions hold:

(i) $(B A)^{\#} B A B=B$;

(ii) $A(A B)^{\#}=(A B)^{\#},(B A)^{\#} A=(B A)^{\#},(A B)^{\#} A=A(B A)^{\#},(B A)^{\#} B=$ $B(A B)^{\#}$;

(iii) $(A B)^{\#} A B A(A B)^{\#}=(A B)^{\#}, A(B A)^{\#}(A B)^{\#} A B=(A B)^{\#}$;

(iv) $(B A)^{\#} B A(A B)^{\#} A=(B A)^{\#}, B(A B)^{\#} A B A=B A$.

Proof. By Lemma 4.1. The proof is similar to Lemma 2.6 of [2].

Theorem 4.3. Suppose $M=\left[\begin{array}{cc}A & A \\ B & O\end{array}\right]$, where $A, B \in \mathbf{R}^{n \times n}, A^{2}=A$, $\operatorname{rank} A=r$, then

(i) $M^{\#}$ exists if and only if $R_{r}(B)=R_{r}(B A B)$;

(ii) If $M^{\#}$ exists, then $M^{\#}=$

$$
\left[\begin{array}{lr}
A-(A B)^{\#}+(A B)^{\#} A-(A B)^{\#} A B A & A+(A B)^{\#} A-(A B)^{\#} A B A \\
(B A)^{\#} B+(B A)^{\#}(A B)^{\#} A B-(B A)^{\#} & -(B A)^{\#}
\end{array}\right]
$$

Proof. (i) It is easy to see that

$$
\begin{gathered}
R_{r}(M)=\left\{\left[\begin{array}{cc}
A & A \\
B & 0
\end{array}\right]\left[\begin{array}{l}
x_{1} \\
x_{2}
\end{array}\right] \mid \forall x=\left[\begin{array}{l}
x_{1} \\
x_{2}
\end{array}\right]\right\}, \text { and } \\
R_{r}\left(M^{2}\right)=\left\{\left[\begin{array}{lr}
A+A B & A \\
B A & B A
\end{array}\right]\left[\begin{array}{l}
y_{1} \\
y_{2}
\end{array}\right] \mid \forall y=\left[\begin{array}{l}
y_{1} \\
y_{2}
\end{array}\right]\right\} .
\end{gathered}
$$

('only if ') Let $x_{1} \in \mathbf{R}^{n}$. It follows from the existence of $M^{\#}$ that $R_{r}(M)=$ $R_{r}\left(M^{2}\right)$. So there exists $y_{1}, y_{2} \in \mathbf{R}^{n}$ such that

$$
\left[\begin{array}{ll}
A & A \\
B & O
\end{array}\right]\left[\begin{array}{l}
x_{1} \\
-x_{1}
\end{array}\right]=\left[\begin{array}{ll}
A & A \\
B & O
\end{array}\right]^{2}\left[\begin{array}{l}
y_{1} \\
y_{2}
\end{array}\right]=\left[\begin{array}{ll}
A+A B & A \\
B A & B A
\end{array}\right]\left[\begin{array}{l}
y_{1} \\
y_{2}
\end{array}\right] .
$$


Then $0=A y_{1}+A y_{2}+A B y_{1}$, and $B x_{1}=B A\left(y_{1}+y_{2}\right)$, so $B x_{1}=-B A B y_{1}$. That is $R_{r}(B) \subseteq R_{r}(B A B) . R_{r}(B) \supseteq R_{r}(B A B)$ is obvious. Hence $R_{r}(B)=R_{r}(B A B)$.

(' if ') Given any $x_{1}, x_{2} \in \mathbf{R}^{n}$. It follows from $R_{r}(B)=R_{r}(B A B)$ that there exists $a \in \mathbf{R}^{n}$ such that $B\left(x_{1}-A\left(x_{1}+x_{2}\right)\right)=B A B a$; that is $B x_{1}=B A\left(x_{1}+x_{2}\right)+B A B a$. Hence

$$
\begin{aligned}
{\left[\begin{array}{ll}
A & A \\
B & O
\end{array}\right]\left[\begin{array}{l}
x_{1} \\
x_{2}
\end{array}\right] } & =\left[\begin{array}{ll}
A\left(x_{1}+x_{2}\right) \\
B x_{1}
\end{array}\right]=\left[\begin{array}{l}
A x_{1}+A x_{2} \\
B A\left(x_{1}+x_{2}\right)+B A B a
\end{array}\right] \\
& =\left[\begin{array}{lr}
0 & A \\
-B A B & B A
\end{array}\right]\left[\begin{array}{l}
-a \\
x_{1}+x_{2}
\end{array}\right]
\end{aligned}
$$

It follows from

$$
\left[\begin{array}{lr}
0 & A \\
-B A B & B A
\end{array}\right]=M^{2}\left[\begin{array}{ll}
I & 0 \\
-I-B & I
\end{array}\right]
$$

that $R_{r}\left(\left[\begin{array}{lr}0 & A \\ -B A B & B A\end{array}\right]\right)=R_{r}\left(M^{2}\right)$. That is $R_{r}(M) \subseteq R_{r}\left(M^{2}\right) . \quad R_{r}\left(M^{2}\right) \subseteq$ $R_{r}(M)$ is obvious. Hence $R_{r}(M)=R_{r}\left(M^{2}\right)$, implying $M^{\#}$ exists.

(ii) By Lemma 4.1 and Lemma 4.2. The proof is similar to Theorem 3.1 of [2] $\mathbf{\square}$

Corollary 4.4. Suppose $M=\left[\begin{array}{cc}A & B \\ A & O\end{array}\right]$, where $A, B \in \mathbf{R}^{n \times n}, A^{2}=A$, $\operatorname{rank} A=r$, then

(i) $M^{\#}$ exists if and only if $R_{l}(B)=R_{l}(B A B)$;

(ii) If $M^{\#}$ exists, then $M^{\#}=$

$$
\left[\begin{array}{lr}
A-(B A)^{\#}+(A B)^{\#} A-(A B)^{\#} A B A & (B A)^{\#} B+(B A)^{\#}(A B)^{\#} A B-(A B)^{\#} \\
A+(A B)^{\#} A-(A B)^{\#} A B A & -(A B)^{\#}
\end{array}\right] .
$$

Proof. It is similar to the proof of Theorem 4.3. $\square$

Theorem 4.5. If $\left[\begin{array}{cc}A & A \\ B & O\end{array}\right]^{\#}$ exists, where $A, B \in \mathbf{R}^{n \times n}, A^{2}=A$, rank $A=r$, then $A B \sim B A$.

Proof. The conclusion is obvious by Corollary 3.2, Theorem 3.6, and Theorem 4.3. प

Remark 4.6. Theorem 4.3, Corollary 4.4, and Theorem 4.5 generalize Theorem 3.1, Corollary 1, and Corollary 2 of [2]. 


\section{REFERENCES}

[1] K.P.S. Bhaskara Rao. The theory of generalized inverses over commutative rings. Taylor and Francis, London and New York, 2002.

[2] Changjiang Bu, Jiemei Zhao, and Jinshan Zheng. Group inverse for a class $2 \times 2$ block matrices over skew fields. Appl. Math. and Comp., 204:45-49, 2008.

[3] S.L. Campbell and C.D. Meyer. Generalized inverses of linear transformations. Dover, New York, 1991 (Originally published: Pitman, London, 1979).

[4] Chongguang Cao. Some results of group inverses for partitioned matrices over skew fields. Heilongjiang Daxue Ziran Kexue Xuebao, 18:5-7, 2001.

[5] Chongguang Cao, Xian Zhang, and Xiaomin Tang. Reverse order law of group inverses of products of two matrices. Appl. Math. and Comput., 158:489-495, 2004.

[6] N. Castro-González and E. Dopazo. Representations of the Drazin inverse of a class block matrices. Linear Algebra Appl., 400:253-269, 2005.

[7] M. Catral, D.D. Olesky, and P. van den Driessche. Group inverses of matrices with path graphs. Electron. J. Linear Algebra, 17:219-233, 2008.

[8] X. Chen and R.E. Hartwig. The group inverse of a triangular matrix. Linear Algebra Appl., 237/238: 97-108, 1996.

[9] P.M. Cohn. Free rings and their relations. Second Edition. London Math. Soc. Monogr. No. 19, Academic Press Inc., London, 1985.

[10] G.H. Golub and C. Greif. On solving block-structured indefinite linear systems. SIAM J. Sci. Comput., 24: 2076-2092, 2003.

[11] Liping Huang. Geometry of matrices over ring. Science Press, Beijing, 2006.

[12] I.C.F. Ipsen. A note on preconditioning nonsymmetric matrices. SIAM J. Sci. Comput., 23: 1050-1051, 2001.

[13] C.D. Meyer and N.J. Rose. The index and Drazin inverse of block triangular matrices. SIAM J. Appl. Math., 33:1-7, 1977.

[14] R. Puystjens. Moore-Penrose inverses for matrices over some Noetherian rings. J. Pure and Appl. Algebra, 31:191-198, 1984.

[15] R. Puystjens and R.E. Hartwig. The group inverse of a companion matrix. Linear Multilinear Algebra, 43:137-150, 1997.

[16] G. Wang, Y. Wei, and S. Qian. Generalized inverse theory and computations. Science Press, Beijing, 2003.

[17] Y. Wei and H. Diao. On group inverse of singular Toeplitz matrices. Linear Algebra Appl., 399: 109-123, 2005. 